\title{
Il confino di polizia de Cesare Pavese
}

\author{
INÉS VALLE MORÁN \\ Universidad Complutense de Madrid \\ ines_va@hotmail.com
}

\section{Resumen}

El presente trabajo tiene una doble finalidad. Por un lado, el análisis de las circunstancias históricas: la detención, el encarcelamiento y el confino di polizia de Cesare Pavese. Por otro lado, se trata de comprender cómo este período histórico (1935-1936) influyó no solo en su vida, sino, y sobre todo, cómo fue significativo en su obra, tanto poética, como narrativa y diarística. El estudio de una serie de textos, entre ellos, el epistolario que mantuvo durante estos años, la novela Il carcere y el nacimiento de Il mestiere di vivere, hace posible, reconstruyendo los múltiples puntos que los unen, entender lo que supuso el confinamento calabro para el escritor turinés. La estrecha interrelación que surge de estos textos entre el tiempo de la vida y el tiempo de la escritura, nos permite, al mismo tiempo, acercarnos a la compleja relación existente entre experiencia vivida y narración en la vida y en la obra pavesiana.

Palabras clave: Cesare Pavese, «confino di polizia», narrativa, Il carcere, Il mestiere di vivere.

\section{The Confino di polizia of Cesare Pavese}

\begin{abstract}
This paper has two central aims. In one hand, to analyze the historical circumstances: the arrest, the detention and the confino di polizia of Cesare Pavese. In the other hand, to understand how this historical period (1935-1936) not only had an influence on his life but above all how it was significant on his poetic, narrative and diaristic work. The analysis of a collection of his works-among them, the letters he exchanged, the novel $\mathrm{Il}$ carcere and the naissance of Il mestiere di vivere- allows us to understand what meant the «confinamento calabro» for the Turin writer, reconstructing all the ties that connect these works. The close linkage that arises from these texts between the art of life and the art of writing, at the same time permits us to approach to the complex relationship that exists between life experience and narrative in the life and work of Pavese.
\end{abstract}

Key words: Cesare Pavese, «confino di polizia», narrative Il carcere, Il mestiere di vivere. 
Sumario: 1. Detención y encarcelamiento. «Il confino a Brancaleone Calabro». 2. Especularidad /contraposición entre narración y vida. Bibliografía.

Valle Morán, Inés. 2011. Il confino di polizia de Cesare Pavese. Cuadernos de Filología Italiana, $\mathrm{n}^{\mathrm{o}}$ extraordinario: págs. 355-367.

\section{Detención y encarcelamiento}

El 15 de mayo de 1935 fueron arrestadas, en la ciudad de Turín, alrededor de 200 personas, entre ellas muchos intelectuales pertenecientes al movimiento Giustizia e Libertà (Pavese 1982: nota p. 232). Pavese y toda la redacción de la revista Cultura, de la cual era Direttore Responsabile pro tempore desde mayo de 1934, resultan detenidos. Junto al escritor, figuran, entre otros, los nombres de Vittorio Foà, Norberto Bobbio, Giulio Einaudi, los Perelli, Carlo Levi, Massimo Mila, Franco Antonicelli y Arrigo Cajumi. Cultura, según la Copia della Relazione del Fiduciario Ovra 373 (Dino Segre, alias Pitigrilli), del 23 de octubre de 1934:

[...] deve essere un ago calamitato sul quale si raduna tutta la limatura di ferro dell'antifascismo cerebrale torinese ${ }^{1}$ (D'Orsi 2005: 143).

Fundada en 1882 por Ruggero Bonghi, retomada y refundada por Cesare De Lollis en 1907, esta revista pasó a ser editada a partir del numero 3 dell'annata XIII (1933) por la recién nacida Casa Einaudi. En otra Relazione di PS (no fechada pero presumiblemente del año 1935), Cultura es definida como la nota rivista; «nota» en cuanto afloraba en aquellos años en las fichas policiales como centro de congregación del antifascismo intelectual de la ciudad de Turín, íntimamente ligado a Giustizia e Libertà, del cual Foà, Massimo Mila y Michele Giuia, «costituiscono il nucleo centrale dle movimento a Torino, intorno a cui si irradiano altri elementi $\rangle^{2}$ (D’Orsi 2005:144). La firma de Pavese aparece por primera vez en el fascículo 11 (noviembre de 1930), con un artículo sobre Sinclair Lewis, premio Nobel de literatura del mismo año. Cuatro años más tarde, el escritor sucederá a Sergio Solmi en la dirección de la publicación, según D’Orsi, porque «è o appare, politicamente il più affidabile, essendo iscritto al PNF» (D’Orsi 2005: 163), en el año 1933.

Pavese es, pues, detenido y conducido a las Carceri Nuove de la ciudad de Turín el 15 de mayo de 1935. En una de las fichas de la prefectura turinesa se lee que «Pavese Cesare Camillo fu Eugenio e Mesturini Consolina», ya en el punto de mira de la policía antes del arresto, a pesar de su inscripción al PNF, posee «sentimenti contrari al Regime» ${ }^{3}$. Interesante para comprender la actuación de la policía y el

\footnotetext{
1 Copia della Relazione del Fiduciario 373, in CSPG, Gl, ga, fasc. 1 (Copia fotostatica; tutti i materiali originali di questo Fondo GL, sono conservati in Archivio di Stato, Torino (D’Orsi 2005:143, nota 2).

2 Relazione s.d. [1935], in CSPG, GL, ga 1, fasc. 1. (D’Orsi 2005: 144, nota 3).

3 Scheda anonima in CSPG, FG, ga, 1. 1 (D’Orsi 2005: 167, nota 77).
} 
arresto del escritor es una scheda riservata, fechada cuatro meses más tarde (el 4 de septiembre, estando ya Pavese en Brancaleone Calabro), en la que se lee que «Laureatosi in belle lettere [...] si fece subito notare ed apprezzare [...]. È giovane colto e di vivida intelligenza». Después de pasar revista a sus comprometedores vínculos, dentro y fuera de Cultura (Leone Ginzburg, «la comunista Pizzardo [...] fidanzata del comunista Spinelli» [D’Orsi 2005: 170]) precisa:

Fu iscritto al PNF nel Luglio 1933, ma gli venne ritirata la tessera in seguito all'arresto. Dal complesso delle sue manifestazioni deve ritenersi che egli abbia richiesta l'iscrizione per mascherare la sua subdola azione favoreggiatrice del movimento antifascista, che fa capo alla setta $a^{4}$ «Giustizia e Libertà» tanto da far presumere che egli stesso vi sia affiliato, essendo stato uno dei più assidui frequentatori dei convegni che si tenevano a Torino fra i componenti il gruppo antifascisti: Antonicelli Franco - Garosci Remo - Levi Alberto - Levi Carlo - Foà Vittorio ecc 5 .

La lectura y el análisis del epistolario que mantuvo con su hermana Maria durante esta primera etapa de encarcelación, primero en Turín, y en un segundo momento, a partir de junio de 1935, en las Carceri di Regina Coeli de Roma, nos permite acercarnos a las impresiones y reflexiones iniciales acerca del porqué de su detención. «Me la caverò ben presto e bene io da me solo. Di questo sono sicuro, perché in questi ultimi mesi non facevo altro che lavorare e non avevo, nemmeno volendo, il tempo di fare prodezze ${ }^{6} \gg$, afirma en la primera de las cartas que envía a su hermana, el día 16 de mayo, tan solo un día después de su arresto, (Pavese 1982: 232). «[...] Continuo a credere che uscirò al più presto» (18 mayo, Pavese 1982: 233), «[...] continuo a non sapere perché sono dentro, ma speriamo che un giorno o l'altro si spieghi» (29 mayo, Pavese 1982: 237). La relación de Pavese «con la prigionia, a dire il vero, non pare del tutto traumatica», afirma Maria Rosa Masoero (Masoero 2000: 979), aunque veremos cómo el tema de la celda, el aislamiento y la prisión será retomado por el escritor e influirá tanto en su narrativa como en su poesía, y aparecerá también en algunos fragmentos del Mestiere di vivere.

El 1 de junio de 1935, Pavese le comunica a su hermana que ha sido informado del cierre de Cultura:

[...] Io ho saputo che è stata soppressa La Cultura e probabilmente tutto questo pasticcio viene di là. Ma non capisco come dirigere una rivista autorizzata, possa

\footnotetext{
${ }^{4}$ [La cursiva es mía].

5 El documento, fechado el 4 de septiembre de 1935, está incluido dentro de ACS, MI, CPC, b. 3790, fasc. «Pavese, Cesare» (D’Orsi 2005: 170).

${ }^{6}$ Todas las cartas escritas por Pavese desde las Carceri Nuove de Turín están timbradas con los sellos «Questura di Torino-Ufficio Politico» y «Carceri Giudiziarie-Torino». En ocasiones, aparece también el siguiente sello: «Visto per censura», al igual que sucederá con las cartas enviadas sucesivamente desde Regina Coeli y desde Brancaleone (Pavese 1982: nota p. 232).
} 
far andare in prigione. Tanto più che si trattava di una rivista letteraria, in cui io avevo l'unico incarico di leggere gli articoli per approvarli (Pavese 1982: 239).

Su principal preocupación en estos primeros días será la publicación de su poemario Lavorare stanca ${ }^{8}$. Una vez trasladado a las Carceri di Regina Coeli en Roma, le pedirá a su hermana que le comunique a Alberto Carocci «che proceda a stampare e a vendere il libro; soltanto, se è ancora in tempo, tolga dal volume l'ultima poesia Una generazione ${ }^{9}$ che, più ci penso, meno mi piace» (Pavese 1982: 242, 14 de junio). A pesar de que el escritor admita el 5 de julio que: «Ho solo un rimpianto (tra i molti): di non poter far poesie. Ma vuol dire che accumulerò il materiale per quando sarò restituito dalla società» (Pavese 1982: 250), había logrado, días antes de escribir estas líneas (el 29 de mayo), componer un poema (un primer esbozo del futuro Poggio Reale, que aparecerá en la edición de enero del 36 de Lavorare Stan$c a$ ). En él transpondrá las sensaciones experimentadas en las Carceri Nuove de Turín a su experiencia en la cárcel napoletana Poggio Reale, lugar al que será conducido durante su traslado hacia el confinamiento (Pavese 1982: 238, nota 2):

Scrivo qui una poesia ${ }^{10}$ che, per non perdere l'abitudine, ho composto, a memoria. [...] Non è molto bella, ma è la prima che compongo a me e quindi mi pare lodevole (Pavese 1982: 238).

${ }^{7}$ [La cursiva es mía] Angelo D’Orsi interpreta esta última frase como evidencia de la «consapevolezza [de Pavese] di essere se non un prestanome, un direttore almeno dimidiato» (D’Orsi 2005: 167). En relación a esto, también debe leerse la carta escrita por el escritor a Giulio Einaudi el 13 de enero de 1935, en la que le comunicaba que «col seguente mese di febbraio ' 35 non ho più intenzione di prestare [la cursiva es mía] il nome come direttore resposabile della rivista La Cultura». La motivación de su cese aparece en una de las cartas enviada a Maria desde Regina Coeli el 26 de julio: «[...] siccome avvenne il sequestro di un numero, per un articolo che spiacque, io diedi subito le dimissioni» (Pavese 1982: 263). Lorenzo Mondo e Italo Calvino (autores de la edición de las Cartas de Pavese 1926-50) afirman, en una de las notas de dicho volumen, que en realidad, la decisión de presentar su dimisión fue debida al cansancio de ser el director nominal de la revista, que de facto era dirigida por Arrigo Cajumi (Pavese 1982: nota 1 264).

${ }^{8}$ El 30 de julio de 1934 el libro estaba ya listo para una última lectura: «Caro Carocci, ecco finalmente il ms. riordinato e tutto. Il titolo, come vedi, ha da essere Lavorare Stanca, motto dell'autore, e, mi pare, nuovo come titolo di versi, in Italia. L'ordine delle poesie è supergiù cronologico, tranne qualche spostamento per chiarire certi filoni d'ispirazione[...]» (carta recogida por Gugliemilnetti en Pavese 2008: VIII).

${ }^{9}$ Los obreros silenciosos que aparecen en Una generazione (que finalmente será incluida en la primera edición del libro de la Edizione Solaria en enero de 1936) hacían temer al escritor que su poesía, de claro contenido político, pudiera ulteriormente agravar su situación (Pavese 1982: nota 1 242). «In prigione / c’è operai silenziosi e qualcuno è già morto. / Nelle strade han coperto le macchie di sangue» (vv. 13-5, Pavese 2008a: 55). Según un comentario de Davide Lajolo, recogido por Calvino, y citado por Guglielminetti (Pavese 2008a: IX), Pavese haría referencia aquí a los obreros «sopra fatti dai fascisti e dalle loro squadracce del '22» (Pavese 2008a:IX, nota 9, 1962: 240).

${ }^{10}$ En dicha carta, Pavese incluirá este poema: «Una breve finestra sul cielo tranquillo / Calma il cuore: qualcuno c'è morto contento. / Non si può avere nuvole e piante, la terra / e anche il cielo; ogni cosa ha da far la sua vita. / Si può dare un'occhiata tranquilla, sapendo / che il cielo ricopre ogni cosa. / I rumori della vita si salgono. L'immobile cielo / vede l'acqua tra i sassi, le case stupite, / le colline e le piante, e raccoglie ogni cosa /nel quadrato leggero. / Compare la nube/ Soda e lieta di quella sua sorte: non vuole spostarsi/ Forse a terra cammina un viandante, che vede la nube / e on sa com'è chiario quel cielo. D'intorno / sono immobili gli alberti e le grandi colline/ e il torrente. / S' invola uno strido di rondine, / ma non tocca quel cielo. Forse un uomo disteso / dentro l'erba, occhi chiusi, ne gode la vita. / Ma quell'uomo non vede altra cosa. Dev'essere morta». 
Un dato curioso, mencionado por el propio Pavese el 8 de julio, es el hecho de que aun siendo la primera vez que es encarcelado, y sin ni siquiera haber pensado en acabar en esta situación, hubiera escrito «una poesia a suo tempo dove si descrivono i sentimenti di un uomo in prigione con la massima fedeltà. Cosa vuol dire l'intuizione! [...] Vero è che ne avevo scritto anche una sulla gioia che si prova a uscir di prigione $[\ldots]^{11} \gg$ (Pavese 1982: 252).

Es finalmente interrogado, y le comunica a su hermana que parece ser que «[...] certe mie conoscenze abbiano combinato, tra loro, chi sa che pasticcio e naturalmente io ci sono coinvolto. Tutti sanno che io non mi sono mai occupato di cose politiche, ma ora pare che le cose politiche si siano occupate di me. Staremo a vedere» (carta fechada el 24 de junio, Pavese 1982: 245). «Afascista inconsapevole?», se pregunta Angelo D'Orsi (D’Orsi 2005: 168). No, en realidad, "afascista consapevole". El desinterés por la política, viejo topos pavesiano, no pocas veces puesto en entredicho (D'Orsi 2005: 169), vuelve a aparecer el 5 de julio, en otra de las misivas enviadas a su hermana: «[...] sappiamo del mio disinteresse per ogni sorta di eresia politica e della mia buona fede. Bisogna convincere Roma» (Pavese 1982: 249).

El 10 de julio, después de casi dos meses de prisión, Pavese es denunciado por la Comisión Central «per l'assegnazione al confino ${ }^{12}$, come pericoloso per l' ordine nazionale « ( 12 de julio 1935; Pavese 1982: 252), ma «per aver svolto a Torino e a Milano attività politica tale da aver nuociuto agli interessi nazionali» (26 de julio

11 Legna verde, escrita en 1934, describía, como bien indica el propio autor, los sentimientos de L'uomo fermo (v. 1), «che è stato in prigione» (vv. 5), lugar en el que «la vita non finiva, ogni giorno filtrava anche il sole: / [...] e il futuro attendeva» (vv. 11-13) (Pavese 2008a: 45). En segundo lugar, hace aquí referencia a otro de sus poemas, incluidos también como el anterior en la edición Solaria de Lavorare stanca, Lagna verde, que comienza con los siguientes versos : «Perché vergogna? Quando uno ha pagato il suo tempo, / se lo lasciano uscire, è perché è come tutti / e ce n'è della gente per strada, che è stata in prigione» (vv. 1-3) (Pavese 2008b: 16).

12 Sería interesante analizar aquí las distintas prácticas de represión política, de internamento y de deportación llevadas a cabo por el régimen fascista. Por falta de espacio, y por sobrepasar los límites de este trabajo, mencionaré brevemente el funcionamiento del confino di polizia, a partir de los estudios de Paola Carucci (2001) y de Carlo Spartaco Capogreco (2006). El confino, introducido por el texto único de las leyes de Pubblica Sicurezza de noviembre de 1926, se convirtió en uno de los elementos clave del sistema coercitivo del régimen fascista, y en el instrumento de represión político más ampliamente adoptado. Se trataba de una medida policial (heredera del domicilio coatto de época liberal), que tenía por objeto la contención del disenso político y social de todos aquellos que pudieran resultar pericolosi per la sicurezza pubblica. Aplicada preventivamente y sustraída a la esfera de la legislación y de la magistratura, esta medida formaba parte de la legislación de policía común, y era ejecutada por vía administrativa (era de hecho la policía y no la magistratura la que decidía si imponer o no el confino). Correspondientes comisiones provinciales, presididas por el prefecto, determinaban el envío al confino (fallo que podía ser recurrido ante las Commissioni d'appello) y cuya duración era variable de uno a cinco años, aunque el límite máximo podía fácilmente ser sobrepasado a través del mecanismo de las reasignaciones. Los opositores fueron deportados a pequeñas localidades (el conocido como confino all'interno) del centro-sur de Italia (incluyendo también Sicilia y Cerdeña), cuya vigilancia estaba bajo el control de los podestà y los carabinieri locales. Sin embargo, aquellos que eran considerados más peligrosos (activistas políticos, obreros, ex-deputados y muchos intelectuales, como Antonio Gramsci) recalaron en islas (conocidas como carceri all'aperto), algunas de las cuales, como la cittadella confinaria de Ventotene, se convirtieron en verdaderos Lager, y en lugares-símbolos de la deportación política italiana. A partir de 1939 llegaron a establecerse, en tierra firme, auténticas colonie confinarie, como la de Pisticci, considerada como el primer campo de concentración fascista. 
1935; Pavese 1982: 261). Circunstancia agravante en la imputación contra Pavese son ciertas relaciones que este mantenía, en particular:

con la nota comunista Pizzardo Battistina ${ }^{13}$, favoreggiando la corrispondenza clandestina con l'antifascista Maffi Bruno, allo scopo di eludere la vigilanza della Polizia e frequentandone assiduamente la casa, ove sovente si parla di teorie socialiste, teorie che la Pizzardo criticava come arretrate in confronto delle proprie più avanzate. / Pertanto, essendosi il nominato Pavese, dimostrato elemento pericoloso per l'ordine Nazionale [sic] per avere svolta attività politica tale da recare nocumento agli interessi nazionali, è stato denunziato alla Commissione Provinciale per l'ammonizione ed il confino di Roma, la quale, nella seduta del 15 luglio scorso, lo ha assegnato al confino di Polizia per la durata di anni tre ${ }^{14}$.

El 17 de julio Pavese envía un brevísimo telegrama : «Assegnato 3 anni confino/ Ignoro destinazione - Cesare- $>\rangle^{15}$. Apela a la Commissione d'Appello, tres días más tarde, a través de un recurso en el que "si richiama al mio memoriale ${ }^{16}$ del 12 e respinge l'accusa in blocco, ammettendo semplicemente innocenti rapporti di amicizia con persone che possono essere sospette» (Pavese 1982: 261). Los principales elementos del recurso serán detallados en una de las últimas cartas ${ }^{17}$ escritas desde Roma, en la que especificará lo siguiente:

[....] se è per la gerenza della Cultura, nego che fosse un giornale antinazionale, anche perché io personalmente ho invitato come collaboratori parecchi camerati, che non vi avrebbero certo scritto, se avessero pensato male della rivista. Qualche firma della rivista, di persone compromesse, era di antichi collaboratori della $\mathrm{Cul}$ tura a cui io non potevo dare un calcio lí su due piedi, ma la tendenza da me impressa alla rivista era di farsi un complesso di collaboratori giovani in regalo. Poi, la Cultura non si occupava di politica. [...] Se la mia incriminazione dipende da certe lettere ricevute da Milano, ripeto che ho ricevuto queste lettere per fare un favore di amicizia a una conoscenza, che non mi risultava più occuparsi di politica, e ignorando il contenuto di queste lettere, che del resto dall'istruttoria mi risultava innocentissimo, concludevo il memoriale riaffermando il mio disinteresse per la politica e le mie occupazioni tutte letterarie e così abbondanti che non mi lasciavano nemmeno il tempo di pensare ad altro (Pavese 1982: 264).

13 En el registro de la casa de la hermana de Pavese, donde este último residía, se encontraron, al parecer, comprometedoras cartas destinadas a la comunista Battistina Pizzardo (Tina), primer gran amor del escritor (Pavese 1982: nota 254).

14 Prefetto di Torino a Ministero dell'Interno, 7 agosto 1935, in ACS, MI, CPC, b. 3790, fasc. «Pavese, Cesare» (D’Orsi 2005: 168-9, nota 84).

15 Carta dirigida a Maria el17 de julio de 1935 (Pavese 1982: 257).

16 Una vez sabido, el día 10 de julio, que ha sido denunciado por la Comisión «per l'assegnazione al confino» (Pavese 1982: 252), el escritor redacta un Memoriale, «dove mi faccio le mie ragioni» (Pavese 1982: 254); cinco días más tarde, recibirá la condena, y es entonces cuando decide redactar dicho ricorso d'appello.

17 Carta a Maria del 26 de julio de 1935. 
Vuelve a emerger, como hemos visto antes, una proclamación expresa de su abierto desinterés hacia lo político, pero no habría que olvidar, como han recogido algunos estudios, que sus intereses culturales no deben ser interpretados como meras evasiones del mundo, fuera de la realidad y de la vida, sino que su excepcional trabajo como organizador cultural denota, en palabras de Giovanni Turi, «una sagacia nient'affato impolitica» (Turi 1989: 171-195). De hecho, su discurso literario constituye, en cierto sentido, un discurso político; existe una dialéctica entre dimensión existencial y dimensión política que, a pesar de que la primera limita a la segunda, emergerá también en algunas páginas del Mestiere di vivere (D’Orsi 2005: 169-70), y le llevará a admitir haberse sentido atrapado «nelle grinfie della storia» ${ }^{18}$.

\section{Il confino a Brancaleone Calabro. Especularidad / contraposición entre narración y vida}

Una vez constado que «per le sue condizioni di salute è ritenuto idoneo a sopportare il regime del confino, significando che il medesimo non risulta ex combattente, non ha benemerenze civili o patriottiche, versa in buone condizioni economiche per cui può mantenersi a proprie spese al luogo di confino» ${ }^{19}$, Pavese recala en tierras calabresas, en el pequeño pueblo de Brancaleone, en provincia de Reggio Calabria.

El período de encarcelamiento y sobre todo los casi 8 meses vividos en el «confino» (agosto 1935-marzo 1936) influirán tanto en la vida como en la obra del escritor. El tiempo de la vida y el tiempo de la escritura, decía Marziano Guglielminetti a la hora de hablar del Mestiere di vivere, no sólo coinciden progresivamente en su diario (Guglielminetti: 2000a: xxxiv), sino que veremos cómo esta interrelación es una de las claves para comprender la producción narrativa, poética y diarística de Pavese durante estos meses. Para ello, hay que partir del análisis de una serie de documentos: la novela $\mathrm{Il}$ carcere, el epistolario de esta época, dos de sus cuentos (Terra d'esilio y Carogne), los poemas redactados en Brancaleone (algunos añadidos en la edición Solaria de Lavorare stanca), y el nacimiento del Mestiere di vive$r e$. Solo su estudio conjunto, la comprensión de los múltiples puntos que los entrelazan, nos permitirá alcanzar una cierta visión global de lo que supuso el «confinamento calabrés» para el escritor de Turín.

La llegada a Brancaleone quedará reflejada en una carta enviada a Maria el día 9 de agosto:

Sono arrivato a Brancaleone domenica 4 nel pomeriggio e tutta la cittadinanza a spasso davanti alla stazione pareva aspettare il criminale che, munito di manette,

${ }_{18}$ Cesare Pavese a G. Vaudagna, 18 de diciembre de 1944, en Pavese (1966a: 740).

19 Questore di Roma a Ministero dell'Interno e Questori di Torino e Cuneo, 16 luglio 1935, (D’Orsi 2005: 16, n. 79, n. 7). 
tra due carabinieri, scendeva con passo fermo, diretto al Municipio. Il viaggio di due giorni, con le manette e la valigia, è stata una impresa di alto turismo. [...] Qui ho trovato una grande accoglienza. Brave persone, abituate a peggio, cercano in tutti i modi di tenermi buono e caro. [...] Qui, sono l'unico confinato. Che siano sporchi è una leggenda. Sono cotti dal sole. Le donne si pettinano in strada, ma viceversa tutti fanno il bagno. Ci sono molti maiali, e le anfore si portano in bilico sulla testa. Imparerò anch'io e un giorno mi guadagnerò la vita nei varietà di Torino $[\ldots]^{20}$.

Pavese entra en contacto con una realidad muy distinta a la suya, con un ambiente remoto que no llegará a aprehender, y en relación al cual se sentirá extraño (Pavese 2008: 29). Pero el confinamiento, al menos en un primer momento (como aflora en las primeras cartas a la hermana), no representa el lugar de la desolación, de la soledad (Masoero 2000: 981). Esta separación forzada, el abandono de Turín y el arribo a estas tierras remotas calabresas, todo ello, llevará al escritor a intentar transformar aquel período y aquel ambiente en un nuevo lugar de trabajo. Poetizar y estudiar: «[...] dei due», apuntará en Il mestiere di vivere, el 29 de diciembre de 1935, «trovo maggiore e più costante conforto nel secondo. Non dimentico però che mi piace studiare in vista sempre del poetare. Ma in fondo il poetare è una ferita sempre aperta, donde si sfoga la buona salute del corpo» (Pavese 2000a: 24).

Entre agosto y diciembre logrará componer 14 poesías $^{21}$, de las cuales, acabarán por fomar parte de la edición Solaria de Lavorare stanca: «otto poesie ${ }^{22}$, frutto di questi ultimi tempi di calma» ${ }^{23}$.

«C'è un parallelo tra questo mio anno $^{24}$ e la considerazione della poesia», subrayaba Pavese en su diario el 22 de frebrero de 1936, y añadía: «Come la sofferenza atroce non l'ho avuto nei grandi momenti (15 maggio, 15 lugio, 4 agosto, 3 febbr. ${ }^{25}$ ) ma in certi lassi furtivi dei periodi intermedî; l'unità del poema non consiste nelle scene-madri, ma nella sottile corrispondenza di tutti gli attimi creativi» (Pavese 2000: 29). Estas primeras páginas de Il mestiere constituyen más bien un ejemplo de journal de l'oeuvre que de un journal intime (Guglielminetti 2000: xxxv). El pro-

20 Carta a Maria del 9 de agosto de 1935 (Pavese 1982: 273-4).

21 El 15 de diciembre de 1935 Pavese escribía estas líneas a su amigo Mario Sturani: «Non capisco poi dove hai letto che io sono uno scoraggiato: che cosa significa questa parola? Non ho bisogno di consigli. Quattro mesi, quattordici poesie, di cui sette superiori a ogni elogio» (Carta recogida en Pavese 2000: 981).

22 Aprovechando la tardanza de la publicación de Lavorare stanca, Pavese incluirá los siguientes poemas: Ulisse, Atavismo, Avventure, Donne appassionate, Luna d'agosto, Terre bruciate, Poggio reale y Paesaggio [VI].

23 Carta a Alberto Carocci, 16 de septiembre de 1935 (Pavese 1982: 283).

24 Hace referencia al período del encarcelamiento y del confinamiento (marzo 35-febrero 36).

25 Vemos aquí uno de los pocos momentos del Mestiere en el que se hace referencia explícita a cuatro fechas que marcarán la vida del autor en estos meses. El 15 de mayo de 1935 es el día del arresto en Turín; el 15 de julio es el día en el que, desde Regina Coeli, comunica a su hermana que a sido "denunciato alla Commissione del Confino» (Pavese 1982: 255); el 4 de agosto es el día de la llegada a Brancaleone; y el 3 de febrero es el día en el que «la Commissione d'Appello ha respinto...il ricorso» (el texto del recurso puede leerse en D. Zucaro, «Carcere e confino. Tre memoriali inediti di Cesare Pavese», in Il Ponte, 1974, pp. 53839, citado en Pavese 2000a: 438, nota 12). 
blema a la hora de enfrentarse a la poesía en esta nueva etapa de su vida radicará en la incapacidad que siente el autor, desde un primer momento, de transformar en poesía la nueva realidad a la que se enfrenta: «Questa sera, sotto le rocce rosse lunari [afirma en Il mestiere di vivere], pensavo come sarebbe di una grande poesia mostrare il dio incarnato di questo luogo. [...] Perché non posso trarre io delle rocce rosse lunari?». La razón residirá en el hecho de que:

esse non riflettono nulla di mio...Se queste rocce fossero in Piemonte, saprei bene però assorbirle in un'immagine e dar loro un significato. [...]. Certamente, [...] dev'essere possibile, anche per me, far poesia su materia non piemontese di sfondo. [...] ciò significa che non sono ancora uscito dalla semplice rielaborazione dell'immagine materialmente rappresentata dai miei legami d'origine con l'ambiente (6 de ocubre de 1935, Pavese 2000: 7).

«Sorpassare Torino», anotará, el 16 de febrero de 1936, significa costruir un «altro mondo», cuyas bases, serán, como siempre, «un periodo ben determinato di dolore e di silenzio» (Pavese 2000a: 25). A Mario Sturani le escribirá una carta al respecto el 27 de noviembre de 1935 en la que le confesará: «Io faccio poesie senza gusto e senz'appetito, e m'accorgo che il mestiere di poeta non serve nemmeno a ammazzare il tempo, perché l'interesse al lavoro viene rarissimo, e troppe sono le ore che è necessario stare tetramente concentrati su un'idea che non c'è. Era già brutto a Torino questo, pensiamo qui» (Pavese 1982: 305).

Esta situación le llevará a madurar la idea de probar el acercamiento al mundo de la prosa. El 6 de octubre de 1935 se preguntará en Il mestiere: «Ma perché, in quel modo che sinora mi sono limitato come per capriccio alla sola poesia in versi, non tento mai un altro genere?» (Pavese 2000a: 8). La dificultad surgirá a la hora de encontrar «un nuovo punto di partenza», dirá el 15 de octubre, pero conoce demasiado bien los obstáculos de esta nueva vía, «cui ho tolto anche la noia tonificante del primo contatto, eppure bisogna percorrerla» (Pavese 2000a: 12-13).

Poco a poco, «la noia, il tedio, la seccaggine, la sgonfia, lo spleen...questa l'incertezza di vita» (Pavese 1982: 299) se van apoderando de él. En la misma carta del 5 de noviembre de 1935, donde se recogen estas impresiones, Pavese le comentará a Adolfo Ruata: «Esercito il più squallido dei passatempi, acchiappo mosche, traduco dal greco, mi astengo di guardare il mare, giro i campi, fumo, tengo uno zibaldone ${ }^{26}$, rileggo la corrispondenza della patria, serbo una inutile castità» (Pavese 1982: 299).

El zilbaldone del que habla: Il mestiere di vivere nace, y no es una mera casualidad, pocos meses después de la llegada de Pavese a Brancaleone. El interés «di questo giornale sarebbe», como recoge Pavese unos años después el 22 de febrero de 1940: «il ripullulare imprevisto di pensieri, di stati concettuali, che di per sé, meccanicamente, segna i grandi filoni della tua vita interna. Di volta in volta cerchi d'intendere che cosa pensi, e solo après coup vai a riscontrarne gli addentellati con giorni antichi $\gg{ }^{27}$ (Pavese 2000a: 175).

\footnotetext{
26 [El subrayado es mío].

27 (Citado por Segre 2000: ivi).
} 
Para él tan solo cuentan, en palabras de Cesare Segre, «i riflessi di questi episodi» (Segre 2000: xviii), tan solo dejará su huella «il ripullulare imprevisto di pensieri, di stati concettuali», apartando cualquier referencia explícita, y es importante subrayar este término, a la realidad histórica vivida. «I grandi filoni della tua vita interna» marcarán efectivamente este zibaldone pavesiano (Pavese 1982: 299), pero influirán al mismo tiempo y de un modo notable en la narrativa y en la poesía producida durante este período.

A pesar de que el precedente político no quede reflejado en estas páginas del diario (para ello, disponemos del contenido del epistolario), es muy importante recalcar cómo, según apunta Marziano Guglielminetti a la hora de hablar de Il mestiere di vivere (pero lo podemos extrapolar a los demás textos anteriormente citados), «il sottinteso di questa scrittura dell'io-creatore sia proprio la condizione forzata di sottrazione alla realtà a lui consueta» (Guglielminetti 2000: xxxv). Esta escritura del "yo-creador", la toma de decisión de comenzar a redactar un «giornale» o «note di diario», en palabras del propio autor (cit. por Segre 2000: xvi), se inicia tan sólo unos meses después de llegar a Brancaleone. Il Secretum professionale constituye la primera parte del diario, aquella que comprende los meses de octubre de 1935febrero de 1936, y la única parte del libro que no cubre un año entero, sino tan solo estos pocos meses hasta la liberación del 15 de marzo. Béatrice Didier, en su trabajo Écrire la Révolution. 1789-1799, resalta los estrechos vínculos existentes entre la encarcelación y la escritura del yo ${ }^{28}$. Lo biográfico desata la escritura del diario, donde se privilegiará la vida interior y el mal de vivir, abandonándose por completo a la reflexión (Segre 2000: xix), y revelándonos, especialmente en estas primeras páginas, los secretos de su profesión: la de poeta (Guglielminetti 2000: xxxvi).

Pero no solo es el episolario el que nos permite integrar en la realidad vivida sentimientos «distillati nel Mestiere di vivere» (Segre 2000: xviii). Es necesario al mismo tiempo complementar el contenido de dichas cartas y del diario con la lectura y el análisis de la novela titulada Il carcere. Creo que es importante insistir en el hecho de que después de todas las dificultades afrontadas por Pavese a la hora de dar cuerpo y forma a su «vena romanzesca» (Masoero 2000: 965), el escritor logre finalmente componer su primera novela, fruto de una situación histórica realmente vivida, y que lo haga a través de una primera prueba narrativa, memorial y retrospectiva (Masoero 2000: 965).

Il carcere fue redactada entre los años 1938 y 1939. La novela nos sugiere, ya desde el título, evidentes enlaces con la experiencia autobiográfica del autor. Diez años fueron necesarios para que dicho libro, cuyo primer título era Memorie di due stagioni $^{29}$, «cosa del passato ${ }^{30}$, «primo tentativo di uscire dal mondo di Lavorare

28 Béatrice Didier, Écrire la Révolution 1789-1799, Paris, Presses Universitaires de France, 1989, citado en Pavese Guglielminetti (2000: xxxvi).

29 Así aparece escrito en el frontispicio del manuscrito autógrafo conservado en el Fondo Einaudi de Turín, en el que se incluyen las fechas de redacción: «27 nov. 1938-16 aprile 1939»; en el centro de dicha página, aparece debajo de tres asteriscos un primer título, tachado con un lápiz azul, «Prigionia (incompiuta)» (Masoero 2000: 964).

${ }^{30}$ Véase a este respecto el pensamiento de Il mestiere di vivere correspondiente al día 1 de enero de 1940 (Pavese 2000a: 169). 
stanca $»^{31}$ y documento di «antifascismo confinario» ${ }^{32}$, viera la luz en 1948 junto con La casa in collina, en el «dittico politico» (Pavese 2000c: xxiii) Prima che il gallo canti.

En Il carcere, «la componente più strettamente autobiografica non trova svolgimento», subraya Laura Nay y Giuseppe Zaccaria, «non nel senso, almeno, di possibili riscontri o verifiche sul piano dei dati auobiografici» (Nay - Zaccaria 2000: 985). Nos encontramos ante una continua descripción de un paisaje interior experimentado por el protagonista de la novela. Stefano percibe la realidad, desde un primer momento, como si viviera «in mezzo a pareti d'aria» (Pavese 2000c: 105). El pueblo, el mar, el cielo, las nubes, su habitación, se convierten para él en «strane realtà»..., [en]... «invisibili pareti d'una cella» (Pavese 2000c: 101), que poco a poco «s'erano connaturate al suo corpo» (Pavese 2000c: 122). «Nessuno si fa casa di una cella, e Stefano si sentiva sempre intorno le pareti invisibili» (Pavese $2000 \mathrm{c}$ : 109). Un continuo ritornello este, el de las pareti invisibili, que recorre toda la novela, y que será apuntado por Franco Antonicelli en una carta a Pavese el 21 de septiembre de $1939^{33}$. «La prigione» quizás, en palabras de Stefano, «non è altro che questo...l'impossibilità di distruggere il tempo» (Pavese 2000c-: 180). Y cada despertar, «era un triste risveglio, come di chi nel carcere abbia dimenticato nel sonno la solitudine. E tra le quattro pareti della chiusa giornata null'altro avveniva» (Pavese $2000 \mathrm{c}$ : 180). «L'angoscia stessa del suo isolamento colorava d'avventura la sua vita» (Pavese 2000c: 122) y las cartas que recibe lo vinculan a «un'esistenza lontana, impenetrabile se non da lui che vi riconosceva se stesso» (Pavese 2000c: 122).

En definitiva, como he tratado de mostrar en las páginas anteriores, las experiencias de Pavese de la cárcel y del confinamiento parecen ser determinantes a la hora de configurar la complejísima relación que establece, a lo largo de toda su obra, entre experiencia vivida y narración. El rechazo explícito inicial a la implicación política se continúa con el intento de alejarse de una realidad de la que se siente ajeno. Y, sin embargo, no puede ser un simple azar que la primera novela que escriba: Il carcere, sea entendida, en palabras del propio autor, como «la rievocazione della mia vita di confino ${ }^{34}$ (Masoero 2000: 978). De este modo, toma forma el que va a ser uno de los rasgos centrales de la obra pavesiana: la especularidad y la contraposición entre vida y obra.

Los estudios realizados sobre $\mathrm{Il}$ mestiere di vivere retoman, una y otra vez, este tema. Las opiniones sobre dicho vínculo, no obstante, no son unánimes, toda vez que el autor adopta posiciones complejas y cambiantes. En opinión de Cesare Segre,

\footnotetext{
${ }^{31}$ Carta a Emilio Cecchi del 17 de enero de 1949 (Pavese 1982: 633).

32 De nuevo se hace aquí referencia a Il mestiere di vivere, 17 nov 1949 (Pavese 2000a: 375).

${ }_{33}$ Carta de Franco Antonicelli a Pavese, en la que se lee: «Forse non sono del tutto in grado di giudicare, perché troppe cose mi sono care come fossero gli stessi ricordi miei [...]. Mi pare che la vita vi circoli con aria viziata e quel paese sia più artefatto che vero. E anche quel ritornello delle «pareti» invisibili che riesce un po' estraneo al lettore e non lo commuove a fondo. Ma sono anche «memorie», è vero. [...] La conclusione? [...] A me è piaciuto non poco, sinceramente» (Masoero 2000: 977).

34 Se hace ahora referencia a la carta dirigida a Giambattista Vicari, fechada en Turín el día 22 de junio de 1941 (Pavese 1982: 398).
} 
en el plano teórico predomina una cesura, como puede verse en lo escrito por Pavese en el diario el 15 de marzo de 1947: «Hai sostenuto che le forme, gli stili, la pagina sono un'altra realtà da quella vissuta. È banale. Ma è una nuova dimensione. Non è che si esprima ${ }^{35}$ niente scrivendo. Si costruisce un'altra realtà, che è parola» (Segre, 2000: xvii, en Pavese 2000 a: 328). El autor retoma con frecuencia el argumento de la contraposición/interacción entre vida y arte (o literatura), oponiendo, por ejemplo, a Dante, Stendhal y Baudelaire, que trabajan: «in un piano chiuso, costruito, avente leggi interne, diverso di quello della vita», a Petrarca, Tolstoi, Verlaine que estarían: «sempre sur l'orlo della confusione di arte e vita» (Segre, 2000: xxvi; Pavese 2000 a: 169-170; 25 de julio de 1940, Pavese 2000 a: 195).

Por su parte, Guglielminetti afirma, en la misma línea, que: «più che di paralelismo si dovrebbe forse parlare di dissociazione.» (Guglielminetti, 2000: xxxvi). En la medida en que la comunión entre poesía, política y amor se unen finalmente bajo el signo del sufrimiento, la disociación se recompone. En este sentido, el gran tema pavesiano sería el de la alternativa al hecho de vivir que está representada por las artes de la palabra: la poesía, en un primer momento, y la narrativa, después.

Finalmente, cabe recodar que Laura Nay y Giuseppe Zaccaria subrayan, por su parte, la existencia de un vínculo estrecho entre novela y autobiografía en la obra del escritor. Una relación que: «egli coglie sia in termini generali nella narrativa contemporanea (la tecnica moderna dei varî personaggi di romanzo che tutti si autobiografanno) sia nella propia esperienza: lo staccare della realtà in racconto in terza persona è un raffinamento di tecnica, ma comincia sempre (?!) col presentare una realtà attraverso un io (autobiografia)» (31 de diciembre de 1937, en Nay - Zaccaria, 2000: 983; Pavese 2000a: 72-73).

\section{Bibliografía}

CAPOGRECO, Carlo. S (2006): I campi del duce. L'internamento civile nell'Italia fascista (1940-1943).Torino, Einaudi.

CARUCCI, Paola (2001): «Il Confino, soggiorno obbligato, internamento: sviluppo della normativa», en I campi di concentramento in Italia. Dall'internamento alla deportazione (1940-1945), a cura di Costantino Di Sante. Milano, F. Angeli, pp. 15-39.

Collotti, Enzo, Sandri, Renato e Sessi, Frediano (2006): Dizionario della Resistenza. Torino, Einaudi.

D’ORSI, Angelo (2005): «L'ago calamitato. Pavese», La Cultura, il Fascismo e l'Antifascismo, en Atti del Convegno Internazionale di Studi, Torino-Santo Stefano Belbo 24-27 ottobre 2001, a cura di Margherita Campanello. Firenze, Olschki, pp. 143-172.

GHINI, Celso - DAL PonT, Adriano (1971): Gli antifascisti al confino 1926-43. Roma, Editori Riuniti.

GuglielminetTI, Marziano (1998): «Introduzione» a Le poesie di Cesare Pavese. Torino, Einaudi, pp. v-xvii.

35 [Resaltado por el propio autor] 
ORECCHIONI, Gianni (2006): I sassi e le ombre: storie di internamento e di confino nell' Italia fascista: Lanciano 1940-3. Roma, Edizioni di Storia e Letteratura.

PAVESE, Cesare (1962): Poesie edite e inedite. Torino, Einaudi.

PAVESE, Cesare (1966): Lettere 1924-1944, vol. I, a cura di Lorenzo Mondo. Torino, Einaudi.

PAVESE, Cesare (1982): Lettere 1926-1950, vol. II, a cura di Italo Calvino (1 ${ }^{\mathrm{a}}$ ed. 1966). Torino, Einaudi.

PAVESE, Cesare (1994a): Racconti. Torino, Einaudi.

PAVESE, Cesare (1994b): Terra d'esilio, en Racconti. Torino, Einaudi, pp. 137-148.

PAVESE, Cesare (1994c): Carogne, en Racconti. Torino, Einaudi, pp. 247-274.

PAVESE, Cesare (2000a): Il mestiere di vivere. Diario 1935-1950, edizione condotta sull'autografo, a cura di Marziano Guglielminetti e Laura Nay, (incluye el ensayo de M. Guglielminetti "La letteratura è una difesa contro le offese della vita". Attraverso Il mestiere di vivere», pp. xxxi-lxii ). Torino, Einaudi.

PAVESE, Cesare (2000b): I capolavori, a cura di Mariarosa Masoero e Giuseppe Zaccaria. Torino, Einaudi .

PAVESE, Cesare (2000c): Tutti i romanzi, a cura di Marziano Guglielminetti. Torino, Einaudi.

PAVESE, Cesare (2008a): Le poesie, a cura di Mariarosa Masoero, "introduzione" di Marziano Guglielminetti. Torino, Einaudi.

SEGRE, Cesare (2000): "introduzione" a Il mestiere di vivere. Torino, Einaudi, v-xxx.

Tranfaglia, Natalino (1996): L'itinerario di Leone Ginzburg. Torino, Bollati Boringhieri.

TURI, Gabriele (1989): Logos e mito: Pavese e la casa editrice Einaudi, in Cesare Pavese oggi. Atti del Convegno Internazionale di Studi, a cura di Giovanna Ioli. Città di San Salvatore Monferrrat, pp. 171-195. 